\title{
An Experimental Evaluation of the Force Requirements for Robotic Mastoidectomy
}

\author{
*Neal P. Dillon, *Louis B. Kratchman, †Mary S. Dietrich, $\$$ Robert F. Labadie, \\ $*$ * Robert J. Webster III, and *Thomas J. Withrow \\ *Department of Mechanical Engineering, †School of Nursing and School of Medicine, and $\$$ Department of \\ Otolaryngology, Vanderbilt University Medical Center, Nashville, Tennessee, U.S.A.
}

\begin{abstract}
Hypothesis: During robotic milling of the temporal bone, forces on the cutting burr may be lowered by choice of cutting parameters. Background: Robotic bone removal systems are used in orthopedic procedures, but they are currently not accurate enough for safe use in otologic surgery. We propose the use of a boneattached milling robot to achieve the required accuracy and speed. To design such a robot and plan its milling trajectories, it is necessary to predict the forces that the robot must exert and withstand under likely cutting conditions.
\end{abstract}

Materials and Methods: We measured forces during bone removal for several surgical burr types, drill angles, depths of cut, cutting velocities, and bone types (cortical/surface bone and mastoid) on human temporal bone specimens.

Results: Lower forces were observed for 5-mm diameter burrs compared with 3-mm burrs for a given bone removal rate. Higher linear cutting velocities and greater cutting depths independently resulted in higher forces. For combinations of velocities and depths that resulted in the same overall bone removal rate, lower forces were observed in parameter sets that combined higher cutting velocities and shallower depths. Lower mean forces and higher variability were observed in the mastoid compared with cortical/surface bone.

Conclusion: Forces during robotic milling of the temporal bone can be predicted from the parameter sets tested in this study. This information can be used to guide the design of a sufficiently rigid and powerful bone-attached milling robot and to plan efficient milling trajectories. To reduce the time of the surgical intervention without creating very large forces, high linear cutting velocities may be combined with shallow depths of cut. Faster and deeper cuts may be used in mastoid bone compared with the cortical bone for a chosen force threshold. Key Words: Mastoidectomy_-Robotic surgery_-Temporal bone milling.

Otol Neurotol 34:e93-e102, 2013.

\section{BACKGROUND}

Robotic systems have been successfully developed for orthopedic bone machining procedures, and several systems are commercially available for joint arthroplasty and resurfacing, including the RIO System (Mako Surgical Corp., Ft. Lauderdale, FL, USA), ROBODOC Surgical System (Curexo Technology Corp., Fremont, CA, USA), and CASPAR (URS Ortho GMBH \& Co. KG, Rastatt, Germany). Bone drilling and bone removal procedures are tasks for which robotics can be especially beneficial because the rigidity of bone enables the procedure to be

Address correspondence and reprint requests to Neal P. Dillon, B.S. Vanderbilt University, PMB 351592, 2301 Vanderbilt Place, Nashville, TN 37235; E-mail: neal.p.dillon@vanderbilt.edu

The authors disclose no conflicts of interest.

Funding received from the National Institutes of Health supported this research. completely planned based on preoperative images, with minimal risk of bone deformation during surgery that could cause deviations from the planned trajectory. In the field of otology, a robotic bone milling system could be beneficial for performing a mastoidectomy, which has been shown to be feasible by Danilchenko et al. (1). Automation of this procedure, which is performed approximately 100,000 times annually (2), could lead to a reduction in operating time and costs and potentially fewer complications (3). Furthermore, because the mastoidectomy is typically a component of a complex surgical procedure, automation of this portion of the surgery could preserve the surgeon's manual dexterity for more delicate, challenging work such as electrode placement in cochlear implantation or tumor removal in acoustic neuroma surgery.

However, the commercial systems mentioned previously are being used for orthopedic procedures and are not accurate enough for otologic surgeries $(4,5)$, which require submillimetric accuracy to avoid critical structures 
embedded and hidden within the bone (e.g., facial nerve, sigmoid sinus, external auditory canal, and carotid artery) (6). To align the drill tip with the target area of the patient and account for any movement during surgery, a tracking system is used to determine the position and orientation of the patient relative to the robot throughout the procedure. This tracking requirement introduces an error in aligning the drill, which is attached to the endeffector of the robot, with the target volume of bone to be removed. One approach to reducing the registration error and relative motion between the robot and the patient is to use a bone-attached robot. The robot's coordinate system can then be registered to the patient's anatomy using fiducial markers in the preoperative images. Automatic segmentation of critical temporal bone anatomy (7-9) can be performed on these images and incorporated into the trajectory planning of a robot to avoid vital structures and optimize the milling path. This bone-attached robotic technique has been shown to be successful for the accuracy required for pedicle screw placement for spinal fusion surgery by the Renaissance Spine Surgery system (Mazor Robotics Ltd., Israel). Additionally, the accuracy required for otologic procedures has been achieved using a compact robot as an automated, stereotactic frame for percutaneous cochlear implantation (10).

To extend the capabilities of bone-attached positioning robots to perform milling with a high level of accuracy and avoid violation of critical structures, it is necessary to consider the forces between the drill tip and bone. Along with the knowledge of the location and dimensional constraints imposed by these structures (6-9), this force data provide the design criteria for a robot that is stiff enough to not have displacements that would cross a given "safety zone" around these structures. To successfully execute a trajectory, the robot's motors must be adequately powered and controlled, and deformations of the robot's frame due to mechanical stresses must be minimized. The robot must be able to exert a specified force in the direction of the cut, while simultaneously rejecting disturbance forces in other directions so that it can maintain the desired trajectory within the specified accuracy. Therefore, knowledge of these forces is an essential component in determining the robot's kinematic design, selecting materials, and choosing actuators. Also, force information for different cutting parameters and bone types may allow the milling trajectory to be optimized. For example, if there is an area that is expected to induce larger forces because of the bone type or drill angle required in that area, specific depths and cutting velocities can be used to ensure that the forces stay below a given threshold. In areas of the milling cavity that yield lower forces, faster and deeper cuts can be specified to minimize the time of the procedure.

Several previous studies have investigated the forces applied in both manual and robotic bone removal (11-15). Arbabtafti et al. performed several force measurement experiments to verify the accuracy of a haptic simulation of bone machining. The authors examined the effects of drill angles, feed rates, and spindle speeds on forces, when milling bovine femurs. The data presented indicate relationships between milling parameters and recorded forces in bone, suggesting a similar pattern may be identified for human temporal bone milling. The authors did not examine the influence of depth of cut, which is known to affect cutting forces and accuracy in industrial milling (16). Plaskos et al. tested forces and specific cutting energies for orthogonal milling in bovine cortical bone for the purpose of modeling and optimizing bone cutting for orthopedic surgery (12). The authors selected cutting parameters similar to those used in clinical practice (very high cutting velocities and shallow depths of cut) and demonstrated that the cutting forces and specific cutting energy are significantly different than at lower speeds. Denis et al. examined the effects of feed per tooth and spindle speed on forces, surface flatness and temperature rise for robotic total knee arthroplasty procedures and concluded that forces increase with feed per tooth (13). Englehardt et al. and Bast et al. examined manual milling forces, temperature, time of procedure, and accuracy of neurosurgeons of different skill levels using bovine scapula specimens $(14,15)$. They then compared robotic milling forces for craniotomies and craniectomies with a robot to that of milling forces of neurosurgeons performing the same procedure and found that the robot was faster and more accurate and resulted in lower forces than the surgeons.

Federspil et al. tested milling forces on human temporal bones and also investigated using a robot to perform a mastoidectomy (17-19). The authors examined some of the parameters required for robotic bone milling by testing milling forces on 2 temporal bone specimens. Different drill rotational speeds, cutting velocities, and various path parameters and burr types were tested, and the authors provided a set of parameters for calvarium bone and mastoid bone $(5 \mathrm{~mm} / \mathrm{s}$ at $30,000 \mathrm{rpm}$ in calvarium and $1 \mathrm{~mm} / \mathrm{s}$ at $30,000 \mathrm{rpm}$ in the mastoid) that best fit within a criterion of $10 \mathrm{~N}$ maximum force and $60^{\circ} \mathrm{C}$ maximum temperature. For a compact, bone-attached robot, physical limitations imposed by the geometry and the specific actuators may require forces lower than $10 \mathrm{~N}$, so it is necessary to characterize the milling forces for a broad range of conditions, including different depths of cut, drill angles, and higher, more clinically relevant drill rotational speeds. It is also important to know the forces associated with nonoptimal milling parameters because optimal milling parameters are not possible in all areas of the milling region. Additionally, to plan an efficient milling trajectory, it is necessary to have relationships between different depth and cutting velocity combinations to determine which combinations result in both lower forces and shorter procedure times. Finally, we believe that more efficient cutting parameters can be used in the mastoid region with respect to the harder bone of other areas of the temporal bone, allowing for faster removal of bone while still maintaining low forces. Therefore, our study focused on examining the milling forces across many temporal bone specimens for a wide range of cutting parameters and different bone types to aid in the design and trajectory optimization of a bone-attached milling robot for performing a mastoidectomy. 


\section{MATERIALS AND METHODS}

To measure milling forces in human temporal bone, an industrial robot was programmed to mill test specimens under a variety of cutting parameters. To simulate the condition of a bone-attached milling robot, which has very little motion between the robot base and the bone, we constructed an apparatus to immobilize temporal bone specimens with respect to the base of the industrial robot (Fig. 1). The specimens were milled using a drill fixed to the end effector of the robot, and force measurements were recorded.

A Mitsubishi RV-3S (Mitsubishi Electric and Electronics, USA, Inc., Cyprus, CA, USA) robot was used for the experiments (20). This robot was chosen for its high repeatability $( \pm 0.02 \mathrm{~mm})$ and ability to achieve the orientations and velocities required for the given parameter ranges in this study; however, it is important to note that the forces measured reflect the cutting parameters and bone type and not this specific robot. An Anspach E-max high-powered electric drill (The Anspach Effort, Inc., Palm Beach Gardens, FL, USA) was fixed to the end-effector of the robot using a custom attachment piece, which also held the tubing used for irrigation of the drill tip and bone. This same model drill is used clinically by surgeons in the Otolaryngology department at Vanderbilt University Medical Center. It can operate at speeds up to 80,000 revolutions per minute and contains an integrated cooling system. An ATI Six-Axis Force/Torque Sensor System (ATI Industrial Automation, Apex, NC, USA) was mounted between the robot end-effector and surgical drill to measure the forces at the drill tip. The force measurements were recorded using a National Instruments DAQ (National Instruments Corp., Austin, TX, USA) at a sampling rate of 10,000 Hz. Defrosted formalin-fixed temporal bone specimens were used for all experimental trials. Formalin-fixed bones have been shown to have similar material properties to fresh cadaver bones and, thus, can be considered as an accurate representation of what will be encountered in vivo (21-24). The specimens were potted in a urethane casting compound to immobilize them during milling with the portion of the bone to be milled positioned 10 to $15 \mathrm{~mm}$ above the top of the urethane. The urethane block was securely fastened to a table approximately 2 feet from the base of the robot.

To obtain accurate and consistent depths of cut, the area of the experimental milling path was premachined by the robot to create a flat surface. The flat surface was machined using the same cutting burr and drill angle as the subsequent experimental run(s), so the exact coordinates of the flat plane in the robot coordinate frame were known. This preparation method took advantage of the high pose repeatability of the robot, and it is reasonable to assume that the error in depth of cut was reduced to the repeatability error of the robot $( \pm 0.02 \mathrm{~mm})$ using this procedure. After the flat plane was created, an experimental cutting pass was made, and the forces were measured in 3 orthogonal directions relative to the position of the force sensor. Based on the angle of the drill, the forces at the surface of the bone could be calculated. The coordinate frame directions relative to the surface of the bone are shown in Figure 2. We programmed the robot to move at a constant depth and cutting velocity for the entire experimental cutting pass, which varied between 14 and $18 \mathrm{~mm}$ based on the restricted amount of bone available to be milled in that particular area of the specimen. The cuts in cortical bone were performed as close to the surface as possible to allow for a large enough flat surface for a given cut (approximately 0.5 to $2.0 \mathrm{~mm}$ deep, depending on the particular specimen). The cuts in the mastoid region were performed approximately 4.0 to $6.0 \mathrm{~mm}$ below the surface of the temporal bone to allow for a long depth of cut that is entirely made up of the trabecular bone.

We performed 4 sets of experiments to measure forces when milling using the parameters listed in Table 1 . In all experiments, the milling was performed using the climb milling approach (i.e., the burr spins such that the flutes contact the bone in the opposite direction as the motion of the burr, see Fig. 2). Because climb milling induces higher forces, these data can be used as design criteria for a robotic system that uses both climb and conventional milling approaches. Along with developing robot design criteria, an objective of this study was to acquire data to aid in optimizing milling parameters. Thus, it was important to first develop a relationship between cutting forces and

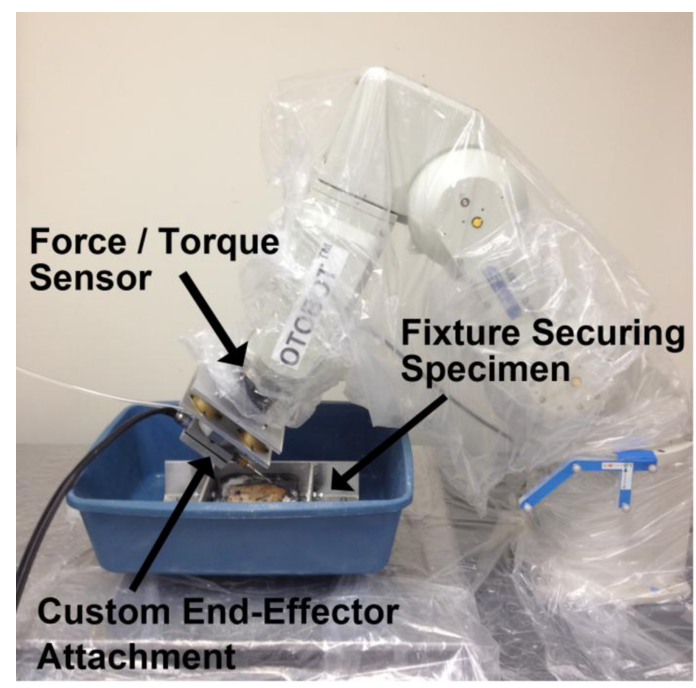

A

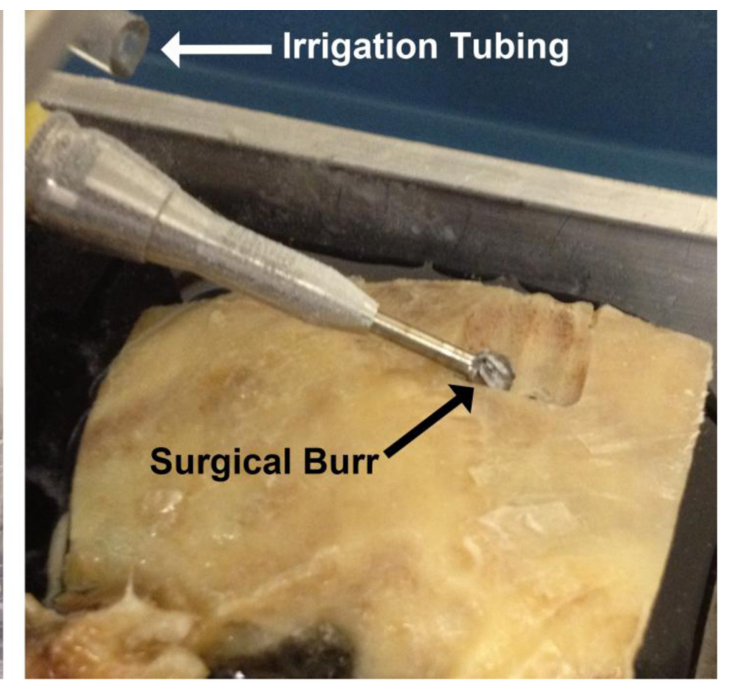

B

FIG. 1. A, Photograph of robotic milling experimental setup. B, Close-up photograph of 5-mm fluted burr milling a path in cortical bone of the temporal bone. 


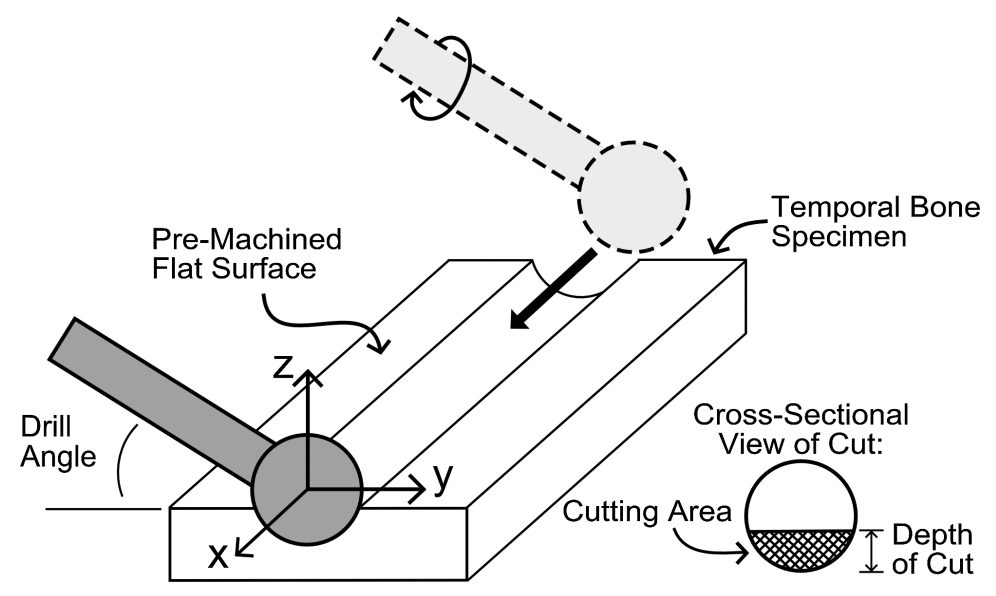

FIG. 2. Schematic of drill moving through the milling path. The surface of each bone was machined to a planar surface before each experimental milling trajectory for accurate determination of the drill angle and depth of cut. The bone removal rate was determined by the cross-sectional area of cut and the cutting velocity.

bone removal rate for the different burr types so that a burr could be chosen that minimizes the overall duration of the milling procedure while maintaining relatively low required forces from the robot. In manual milling procedures, the surgeon will often change burr types for different areas of the milling cavity. However, in a robotic system, it is desirable to use a single burr if possible to minimize the overall time of the procedure and avoid complications related to re-registering the images of the patient's anatomy to the robot's coordinate frame after the burr is changed. It is also necessary to compare different cutting angles because certain areas of the milling cavity permit different drill orientations. Thus, it is important to identify any differences in forces for the various orientations. Next, it is important to compare the forces at various depths of cut and cutting velocities. Together with the burr size, these parameters determine the bone removal rate and, in turn, the duration of a procedure. In solid bone, the bone removal rate is equivalent to the crosssectional area of the burr engaged with the bone multiplied by the linear cutting velocity as shown in Figure 2. The bone removal rate has units of volume of bone over time. In addition to choosing a bone removal rate, it may be possible to choose the combination of depth and cutting velocity for a specific rate that yields lower forces. Therefore, the different depths and velocities tested were selected to allow for comparison of each parameter individually, comparison of different bone removal rates, and comparison of parameter combinations for a specific removal rate. Finally, because a mastoidectomy requires the removal of both cortical and trabecular bone, these 2 bone types were tested and compared. Because of the variability in both hardness and porosity in bone between various specimens, each trial for a given set of cutting parameters was performed in a different specimen, and in most cases, a given specimen was used for 1 trial of each set of parameters being compared.

\section{Comparison of Cutting Burr Types}

We selected 3 cutting burrs that are used frequently in mastoidectomy procedures for comparison-a 5-mm fluted burr, a 3-mm fluted burr, and a 3-mm diamond burr. Fluted burrs have cutting flanges, which remove tissue by cutting radially from the center of the bit, whereas diamond burrs have industrial diamonds embedded on their surface to radially abrade tissue. They were tested in cortical bone at a fixed angle of 20 degrees and drill speed of 80,000 RPM under 2 different combinations of cutting velocity and depth of cut. The velocities and depths were chosen such that the bone removal rates were the same for the 3and 5-mm burrs (i.e., the 3-mm burr must cut faster at a given depth or deeper at a given velocity to remove the same amount of bone as the larger burr). In one of the bone removal rates, the cutting velocity for the 3-and 5-mm burrs were equal, and in the other bone removal rate, the depths were equal.

\section{Comparison of Drill Angles}

In the next experiment, drill angles of 20, 40, 60, and 90 degrees were tested. The drill angle is measured relative to the surface of the bone being cut. In this experimental setup, the angle is measured from the planar surface of the bone (Fig. 2). Lower angles use more of the side of the burr, whereas higher angles use more of the tip of the burr. For all of these trials, which were performed in cortical bone, a 5-mm fluted burr was used along with a spindle speed of $80,000 \mathrm{RPM}$, cutting velocity of $2 \mathrm{~mm} / \mathrm{s}$ and depth of $1 \mathrm{~mm}$.

\section{Comparison of Various Depths of Cut and Cutting Velocities}

To test different combinations of depths of cut and cutting velocities, depths were chosen between 0.5 and $1.5 \mathrm{~mm}$, and cutting velocities were chosen between 1 and $8 \mathrm{~mm} / \mathrm{s}$ for a total of 14 different combinations (Table 2). The depth of cut and velocity combinations tested were chosen in part to allow for comparison of different depth and cutting velocity combinations that resulted in the same overall bone removal rate. For all of the trials in this study, a 5-mm fluted burr was used at an angle of 20 degrees and a drill spindle speed of 80,000 RPM. Again, cortical bone in the temporal bone of a human skull was used for all trials.

TABLE 1. Cutting parameters and ranges tested in this study

\begin{tabular}{lc}
\hline Cutting parameter & Range tested \\
\hline Burr type & $3 \mathrm{~mm}$ fluted, $5 \mathrm{~mm}$ fluted, $3-\mathrm{mm}$ diamond \\
Drill angle & $20-90$ degrees \\
Depth of cut & $0.62-1.64 \mathrm{~mm}$ \\
Cutting velocity & $1-8 \mathrm{~mm} / \mathrm{s}$ \\
Drill spindle speed & $80,000 \mathrm{RPM}$ (held constant) \\
\hline
\end{tabular}


TABLE 2. Depth/velocity combinations tested and the corresponding bone removal rate for each combination

\begin{tabular}{lcc}
\hline Depth $(\mathrm{mm})$ & Velocity $(\mathrm{mm} / \mathrm{s})$ & $\begin{array}{c}\text { Corresponding bone } \\
\text { removal rate }\left(\mathrm{mm}^{3} / \mathrm{s}\right)\end{array}$ \\
\hline 0.62 & $2.0,4.0,8.0$ & $2.8,5.6,11.2$ \\
1.00 & $1.0,2.0,4.0,6.0$ & $2.8,5.6,11.2,16.8$ \\
1.33 & $1.0,2.0,4.0,5.35$ & $4.2,8.4,16.8,22.4$ \\
1.64 & $1.0,2.0,4.0$ & $5.6,11.2,22.4$ \\
\hline
\end{tabular}

Each row shows all of the velocities tested for that particular depth.

\section{Comparison of Different Bone Types}

In the final experiment, a subset of the parameters used previously in cortical bone was tested in the trabecular bone in the mastoid region (Fig. 3). Three depth/velocity combinations were chosen to allow for comparisons at multiple sets of cutting parameters. Because there is more variation in the mastoid within a single specimen than in cortical bone, 2 trials for each mastoid specimen were performed, compared with 1 trial per specimen in the cortical bone.

In each trial for all 4 of the experiments discussed previously, only the force measurements after the burr was completely engaged in the bone were considered; the "transient" portion of the data, as the forces rose from zero to their relatively "steadystate" value, was removed. Figure 4 shows an example of the recorded forces for a typical trial, including the transient rise in forces. Once steady-state was achieved, mean force values for each condition over the remaining portion of the trial were generated. Comparisons of 2 sets of mean force values (e.g., \#\# versus \#\#) were conducted using independent samples $t$ tests. Comparisons of more than 2 sets of mean force values (e.g., \#\# versus \#\# versus \#\#) were initially tested using a 1-way analysis of variance. If there was a statistically significant difference among the means, post hoc pairwise comparisons were conducted to determine which specific pairs of differences were accounting for the overall finding. If the assumption of homogeneity of means was met, Bonferroni adjusted post hoc tests were used; if not, Dunnett $C$ tests were used. Given the preliminary nature of this work, other than for the post hoc tests, no adjustment to the overall alpha of 0.05 was used. In other words, an alpha of $0.05(p<0.05)$ was used to determine statistical significance.

\section{RESULTS}

\section{Comparison of Cutting Burr Types}

In the first experiment, the 5-mm fluted burr achieved bone removal rates with lower forces than both the 3-mm fluted and the 3-mm diamond burrs (Fig. 5). For a bone removal rate of $4.1 \mathrm{~mm}^{3} / \mathrm{s}$, the mean force exerted on the 5-mm fluted burr was $0.75 \mathrm{~N}( \pm 0.28 \mathrm{~N})$ lower than the 3 -mm fluted burr $(p=0.041)$ and $1.16 \mathrm{~N}( \pm 0.28 \mathrm{~N})$ lower than the $3-\mathrm{mm}$ diamond burr $(p=0.001)$. For a bone removal rate of $8.4 \mathrm{~mm}^{3} / \mathrm{s}$, the mean force exerted on the 5-mm fluted burr was $0.90 \mathrm{~N}( \pm 0.27 \mathrm{~N})$ less than the 3-mm fluted burr $(p=0.009)$ and $1.37 \mathrm{~N}( \pm 0.27 \mathrm{~N})$ lower than the $3-\mathrm{mm}$ diamond burr $(p<0.001)$. The difference in cutting forces between the 2 different 3-mm burr styles was not statistically significant. Because the forces were lower for the 5-mm fluted burr and the larger burr allows for more depth/velocity combinations for a given bone removal rate, allowing more flexibility in trajectory planning, the 5-mm fluted burr was chosen to be used for the other experiments.

\section{Comparison of Drill Angles}

In the second experiment, four drill angles (20, 40, 60, and 90 degrees) were compared using the 5-mm fluted burr and constant spindle speed, cutting depth and linear cutting velocity. The mean and maximum forces recorded for each trial of the 4 angles tested are given in the Appendix. There was not a statistically significant difference in mean forces between these angles; however, the maximum forces measured were higher for greater cutting angles. Figure 6 shows an example of the high spikes in forces for greater drilling angles.
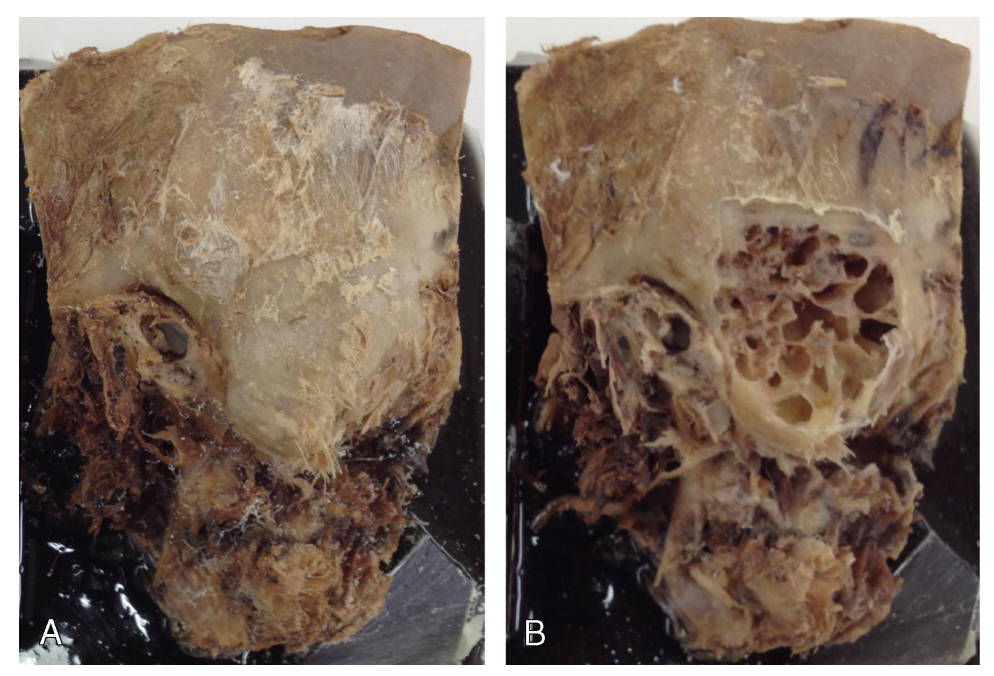

FIG. 3. A, Photograph of temporal bone specimen before milling. $B$, Specimen after experimental trials showing the trabecular bone of the mastoid tip. 


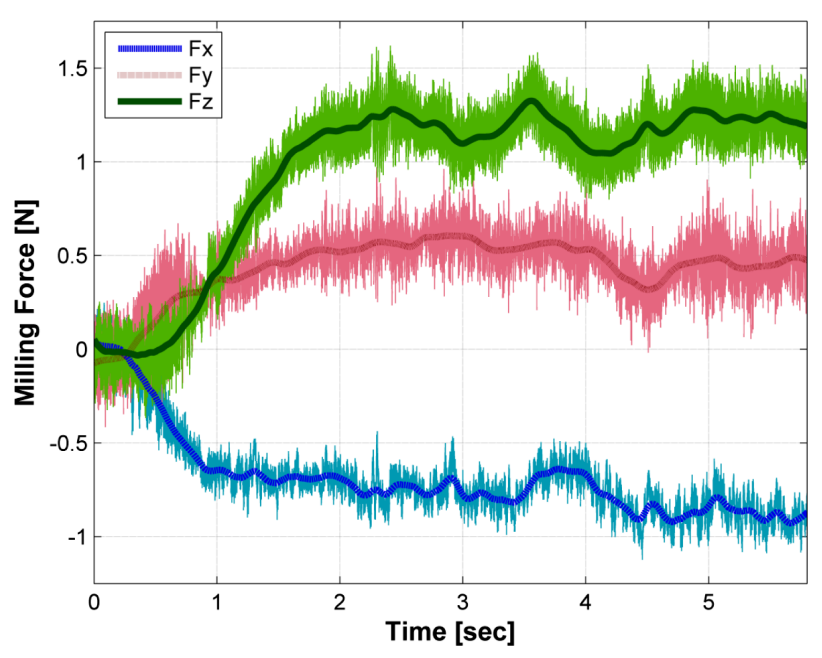

FIG. 4. Typical plot of experimental data in cortical bone. This plot shows a trial using a 3-mm fluted burr at an angle of 20 degrees relative to the bone, a depth of cut of $1.00 \mathrm{~mm}$, and a velocity of $2.0 \mathrm{~mm} / \mathrm{s}$. The data have been transformed to the coordinate frame relative to the bone, as shown in Figure 2. For each force direction $(\mathrm{x}, \mathrm{y}, \mathrm{z})$, the dark lines represent a moving average of the data and the light-colored lines represent the instantaneous measurements.

\section{Comparison of Various Depths of Cut and Cutting Velocities}

As seen in Figures 7 and 8, the mean cutting force increased as depth of cut and cutting velocity increased. This was true for each of the depths of cut tested and each of the cutting velocities tested. The mean cutting force increased more sharply for increases in depth of cut compared with increases in cutting velocity. See Appendix A for more detailed results of each of the depth/velocity combinations as well as the statistical comparisons of the results shown in Figures 7 and 8. Five of the bone removal rates included in the experiment were tested with multiple depth/velocity combinations. In each of these cases

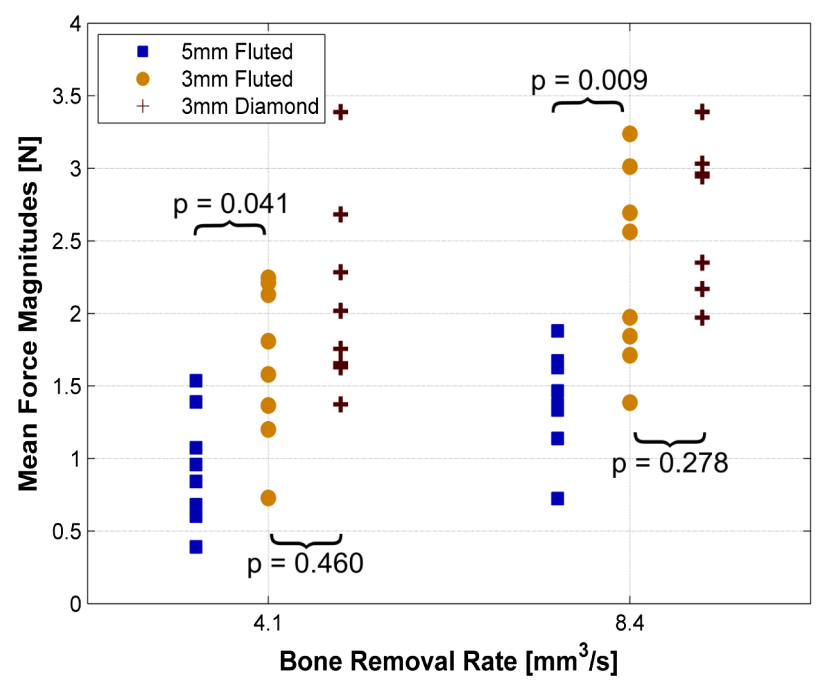

FIG. 5. Comparison of mean force magnitude for the 5-mm fluted, 3-mm fluted, and 3-mm diamond burrs. For both bone removal rates, the 5-mm fluted burr had lower mean forces.

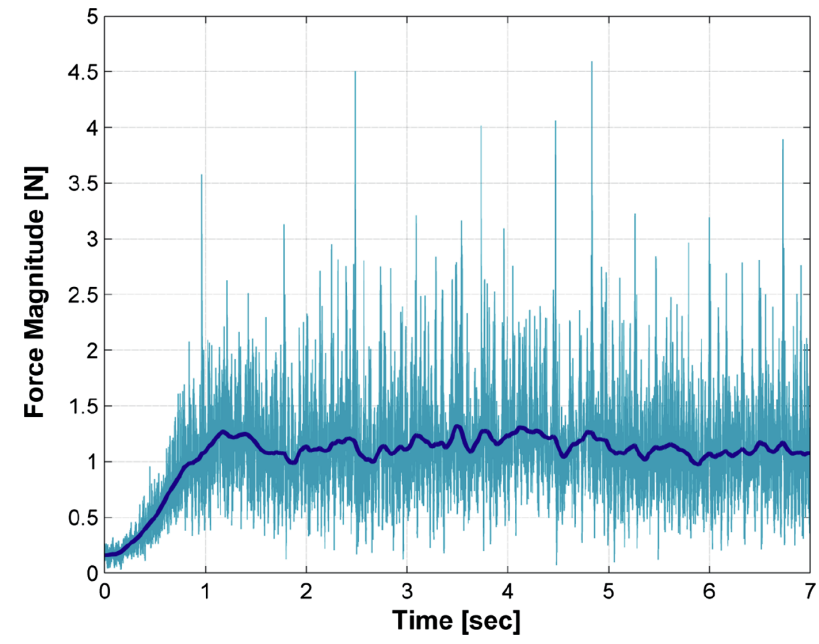

FIG. 6. Plot of experimental data using drill angle of 90 degrees relative to the bone surface. The mean force (represented by the dark blue line) is approximately $1.1 \mathrm{~N}$; however, the force increases notably at several points along the cutting path, reaching forces of up to 4.6 N. The light blue data represents instantaneous measurements.

(Table 3), the mean force was lower for the settings that had a shallower depth of cut and faster cutting velocity.

\section{Comparison of Different Bone Types}

In the final portion of this study, the forces arising in the trabecular bone within the mastoid were significantly lower than in cortical bone for the 2 cases tested using higher depths and velocities. Additionally, the variability in force was higher for the trabecular bone in both cases. For a depth of $1.0 \mathrm{~mm}$ and velocity of $4.0 \mathrm{~mm} / \mathrm{s}$, the mean force was $1.32 \mathrm{~N}$ for the cortical bone with a coefficient of variance of 0.13 compared with a mean force of 0.86 $\mathrm{N}$ for trabecular bone with a coefficient of variance of 0.56 ( $p<0.001$ for comparison of mean forces). For a depth of $1.64 \mathrm{~mm}$ and velocity of $4.0 \mathrm{~mm} / \mathrm{s}$, the mean force was

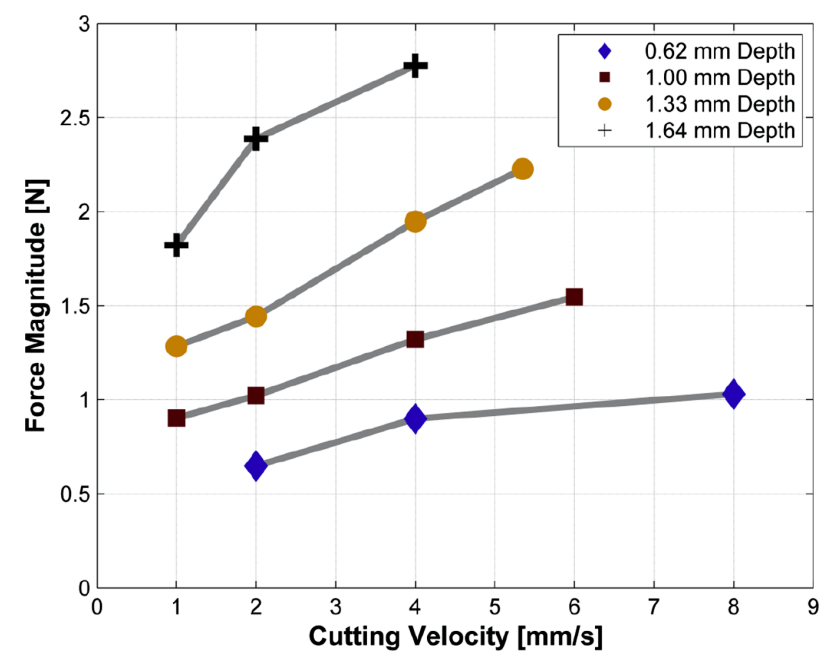

FIG. 7. Comparison of mean force magnitudes for various linear cutting velocities at 4 different depths of cut. 


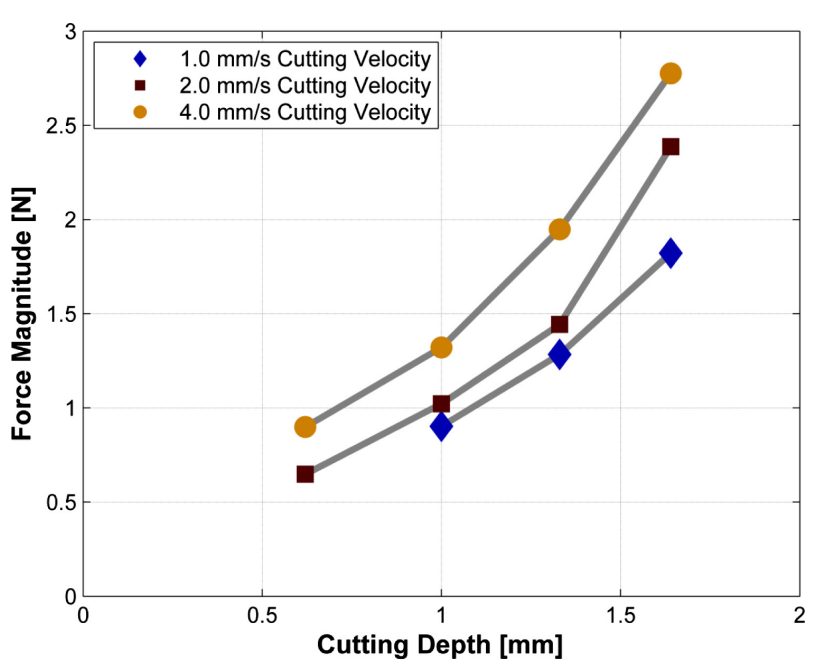

FIG. 8. Comparison of mean force magnitudes for various cutting depths at 3 different linear cutting velocities.

$2.77 \mathrm{~N}$ for the cortical bone with a coefficient of variance of 0.12 compared with a mean force of $1.44 \mathrm{~N}$ for the trabecular bone with a coefficient of variance of 0.44 ( $p=$ 0.016 for comparison of mean forces). For the shallower/ slower set of parameters $(1.0 \mathrm{~mm}, 1.0 \mathrm{~mm} / \mathrm{s})$, the forces for both bone types were very low, so there was not a statistically significant difference in mean force values ( $p=$ 0.077). Even within a single specimen, the forces can vary considerably between cutting paths, so 2 trials for each set of parameters were performed in each of the 8 specimens. Figure 9 shows the variability along a cutting path in the mastoid as well as the potential difference in both magnitude and direction of force from one path to another.

\section{CONCLUSION}

Because forces at the drill tip are transferred up the shaft and result in deflection of the robot, these forces

TABLE 3. Comparison of different depth/velocity combinations for a given bone removal rate

\begin{tabular}{lccccc}
\hline $\begin{array}{l}\text { Bone removal } \\
\text { rate }\left(\mathrm{mm}^{3} / \mathrm{s}\right)\end{array}$ & $\begin{array}{c}\text { Depth/ } \\
\text { Velocity } \\
1(\mathrm{~mm} / \mathrm{s})\end{array}$ & $\begin{array}{c}\text { Depth/ } \\
\text { Velocity } \\
2(\mathrm{~mm} / \mathrm{s})\end{array}$ & $\begin{array}{c}\text { Mean force } \\
\text { difference } \\
(1-2)(\mathrm{N})\end{array}$ & $\begin{array}{c}\text { Standard } \\
\text { error }(\mathrm{N})\end{array}$ & $p$ \\
\hline 2.8 & $0.62,2.0$ & $1.0,1.0$ & -0.26 & 0.11 & 0.039 \\
5.6 & $0.62,4.0$ & $1.0,2.0$ & -0.12 & 0.09 & 0.596 \\
& $0.62,4.0$ & $1.64,1.0$ & -0.92 & 0.09 & $<0.001$ \\
& $1.0,2.0$ & $1.64,1.0$ & -0.80 & 0.09 & $<0.001$ \\
11.2 & $0.62,8.0$ & $1.0,4.0$ & -0.29 & 0.07 & $<0.05^{a}$ \\
& $0.62,8.0$ & $1.64,2.0$ & -1.36 & 0.18 & $<0.05^{a}$ \\
& $1.0,4.0$ & $1.64,2.0$ & -1.07 & 0.18 & $<0.05^{a}$ \\
16.8 & $1.0,6.0$ & $1.33,4.0$ & -0.40 & 0.16 & 0.028 \\
22.4 & $1.33,5.35$ & $1.64,4.0$ & -0.55 & 0.22 & 0.058 \\
\hline
\end{tabular}

All trials were performed in cortical bone with a 5-mm fluted burr, at a 20-degree drill angle, and a drill spindle speed of 80,000 RPM. "Depth/ Velocity Set 1" and "Depth/Velocity Set 2" are the 2 depth/velocity combinations being compared. In all cases, lower forces were achieved for a given bone removal rate with lower cutting depths and higher cutting velocities.

${ }^{a}$ Because of inhomogeneous variances, Dunnett $\mathrm{C}$ test was used, and exact $p$ value not given.
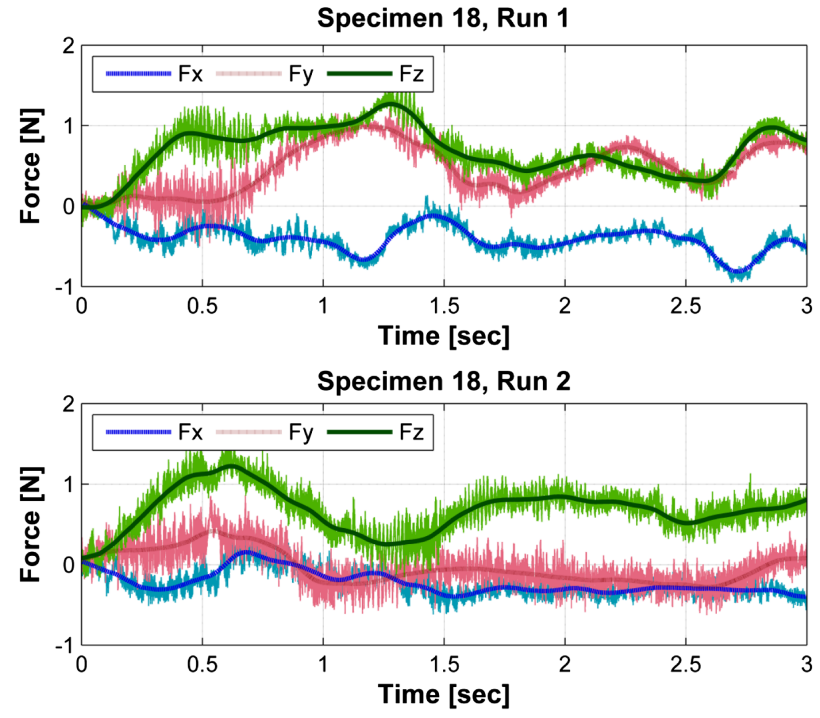

FIG. 9. Examples of milling forces in the mastoid. Lower overall forces were observed in the mastoid than in cortical bone, although both the direction and magnitude of the force showed greater variance than in the cortical bone. For each force direction ( $x, y, z)$, the dark lines represent a moving average of the data, and the lightcolored lines represent the instantaneous measurements.

have a direct effect on the accuracy of the robot. Therefore, it is necessary to incorporate this force informationalong with the necessary robot workspace, required accuracy and size constraints - in the design of a bone-attached robot for mastoidectomy and other skull-based surgeries. In addition to its use in the design process, the force data presented in this study can be used to optimize the milling trajectory such that the forces at the drill tip are minimized. Based on the forces observed in this study, it is reasonable to assume that by incorporating this data into the design and trajectory planning of a robotic system, the procedure can be completed faster by a robot than a human.

The lower force using the 5-mm burr compared with the 3-mm burr at similar bone removal rates allows for faster removal of the target bone volume. Compact robots are inherently limited in the force that they can produce and withstand; thus, it is desirable to use the larger burr when possible to achieve a given bone removal rate. However, geometrical constraints of the milling cavity and the critical anatomy within the cavity do not always permit larger burr sizes, so these constraints must be balanced with the expected forces when choosing the appropriate burr and cutting/trajectory specifications.

The mean forces at the drill tip do not seem to be significantly affected by the drill angle. However, relatively large transient forces were observed at greater drill angles, suggesting that the angle of the burr relative to the cutting surface should be minimized when possible. While milling larger areas such as the layer of cortical bone and the outer portion of the mastoidectomy, it is possible to cut at very small angles, thus using the side portion of the burr for the majority of the cut. However, for the 
portions of the trajectory that are deeper in the skull, it is not always possible to cut at optimal angles. In these areas, the cutting velocity and/or depth of cut must be reduced to avoid large increases in force that could affect the accuracy of the robot.

Lower forces were observed for the same bone removal rate for parameter combinations that used shallower depths and higher cutting velocities compared with deeper cuts at lower velocities. This information can be applied to the design of the robot by specifying actuators and gearing that allow for higher end-effector velocities. Furthermore, the time for a robotic procedure can be reduced without increasing forces on the robot-and thus impacting the accuracy - by limiting the depth of cut and increasing the cutting velocity. Although none of the cutting parameters tested in this experiment resulted in forces higher than $5 \mathrm{~N}$, higher values should be anticipated in the design of the robot to account for potential spikes in forces as seen in the angle comparison trials at higher angles and as a general factor of safety. This factor of safety will also account for any differences between the experimental forces measured in formalin-fixed bones and forces in vivo.

The different forces arising in cortical bone compared with the pneumatized bone of the mastoid can also be applied to planning the milling trajectory. The robot should be designed to withstand the higher forces that occur while milling cortical bone but specifying different cutting parameters in the mastoid region allows for optimal path planning. However, the cumulative density of bone in the mastoid - accounting for both bone and aircan differ significantly from patient to patient. In general, milling in well-pneumatized bone results in lower forces for a given depth and cutting velocity as there is a lower amount of bone to be removed overall. However, because of the irregularity of the bone and air patterns, the burr can be in contact with different quantities of bone throughout the cut leading to more variability. Even within a single specimen, the forces can vary significantly between cutting paths. Thus, a robotic system should use force control to allow for altering the cutting parameters (i.e., cutting velocity) based on the irregularity of bone throughout its cutting path. Additionally, a topic of future work in this area could focus on developing a relationship between milling forces and bone density in the mastoid, air pocket sizes and patterns to allow for further improvements of the planned trajectory and patient-specific cutting parameter ranges.

\section{REFERENCES}

1. Danilchenko A, Balachandran R, Toennis JL, et al. Robotic mastoidectomy. Otol Neurotol 2011;32:11-6.

2. French LC, Dietrich MS, Labadie RF. An estimate of the number of mastoidectomy procedures performed annually in the United States. Ear Nose Throat J 2008;87:267-70.

3. Plinkert B, Plinkert PK. Robotics in skull base surgery. Int Congr Ser 2001;1230:138-42.
4. Nishihara N, Sugano N, Nishii T, et al. Clinical accuracy evaluation of femoral canal preparation using the ROBODOC system. J Orthop Sci 2004;9:452-61.

5. Schneider J, Kalender W. Geometrical accuracy in robotassisted total hip replacement surgery. Comput Aided Surg 2003; 3:135-45.

6. Labadie RF, Majdani O, Fitzpatrick JM. Image-guided technique in neurotology. Otolaryngol Clin N Am 2007;40:611-24.

7. Noble JH, Dawant BM, Warren RM, Majdani O, Labadie RF. Automatic identification and 3-D rendering of temporal bone anatomy. Otol Neurotol 2009;30:436-42.

8. Noble JH, Warren FM, Labadie RF, Dawant BM. Automatic segmentation of the facial nerve and chorda tympani in CT images using spatially dependent feature values. Med Phys 2008;35: 5375-84.

9. Labadie RF, Mitchell J, Balachandran R, Fitzpatrick JM. Customized, rapid-production micro-stereotactic table for surgical targeting: description of concept and in-vitro validation. Int J Comput Assist Radiol Surg 2009;4:273-80.

10. Kratchman LB, Blachon GS, Withrow TJ, Balachandran R, Labadie RF, Webster RJ. Design of a bone-attached parallel robot for percutaneous cochlear implantation. IEEE Trans Biomed Eng 2011; 58:2904-10.

11. Arbabtafti M, Moghaddam M, Nahvi A, Mahvash M, Richardson B, Shirinzadeh B. Physics-based haptic simulation of bone machining. IEEE Transactions on Haptics 2011;4:39-50.

12. Plaskos C, Hodgson AJ, Cinquin P. Modelling and optimization of bone-cutting forces in orthopaedic surgery. In: Medical Image Computing and Computer-Assisted Intervention, vol. 2878. Berlin, Germany: Springer, 2003;254-61

13. Denis K, Van Ham G, Vander Sloten J, et al. Influence of bone milling parameters on the temperature rise, milling forces and surface flatness in view of robot-assisted total knee arthroplasty. Int Congr Ser 2001;1230:300-6.

14. Engelhardt M, Bast P, Lauer W, Rohde V, Schmieder K, Radermacher K. Manual vs robotic milling parameters for development of new robotic system in cranial surgery. Int Cong Ser 2004;1268:533-8.

15. Bast P, Engelhardt M, Lauer W, Schmieder K, Rohde V, Radermacher K. Identification of milling parameters for manual cutting of bicortical bone structures. Comput Aided Surg 2003;5: 257-63.

16. Kalpakjian S, Schmid SR. Manufacturing Engineering and Technology, 5th ed. Upper Saddle River, NJ: Pearson Prentice Hall, 2006:607-26.

17. Federspil PA, Geisthoff UW, Henrich D, Plinkert PK. Development of the first force-controlled robot for otoneurosurgery. $L a$ ryngoscope 2003;113:465-71.

18. Federspil PA, Plinkert B, Plinkert PK. Experimental robotic milling in skull-base surgery. Comput Aided Surg 2003;8:42-8.

19. Federspil PA, Plinkert PK. Robotic surgery in otorhinolaryngology. Otolaryngologia Polska 2004;1:237-42. LVIII.

20. Mitsubishi Electric Industrial Automation. Technical Manual: RV3SB/3SJB Series Standard Specifications Manual (CR1B-571/ CR2B-574 Controller). Available at: http://www.meau.com/eprise/ main/sites/public/Downloads/Manuals/default. Published 10/12/2009. Accessed June 1, 2012

21. Currey JD, Brear K, Zioupos P, Reilly GC. Effect of formaldehyde fixation on some mechanical properties of bovine bone. Biomaterials 1995;16:1267-71.

22. Van Haaren EH, Van der Zwaard BC, Van der Veen AJ, Heyligers IC, Wuisman PIJM, Smit TH. Effect of long-term preservation on the mechanical properties of cortical bone in goats. Acta orthopaedica 2008;79:708-16.

23. Topp T, Müller T, Huss S, et al. Embalmed and fresh frozen human bones in orthopedic cadaveric studies: which bone is authentic and feasible? Acta Orthopaedica 2012;83:543-7.

24. Zech S, Goesling T, Hankemeier S, et al. Differences in the mechanical properties of calcaneal artificial specimens, fresh frozen specimens, and embalmed specimens in experimental testing. Foot Ankle Int 2006;27:1126-36. 


\section{APPENDIX}

The following tables provide results for the comparison of forces for the 4 different drill angles, each of the 14 combinations tested in the experiment comparing various depths and cutting velocities, as well as the statistical comparison of mean force magnitude for the different velocities tested at a constant depth and the different depths tested at a constant velocity.

\section{APPENDIX TABLES}

TABLE A1. Force magnitude data (in Newtons) for all trials for each drill angle tested. All trials were performed in cortical bone with a $5 \mathrm{~mm}$ fluted burr, a depth of cut of $1 \mathrm{~mm}$, a cutting velocity of $2 \mathrm{~mm} / \mathrm{s}$, and a drill spindle speed of 80,000 RPM.

\begin{tabular}{|c|c|c|c|c|c|c|c|c|}
\hline \multirow[b]{2}{*}{ Trial \# } & \multicolumn{2}{|c|}{ 20-degree drill angle } & \multicolumn{2}{|c|}{ 40-degree drill angle } & \multicolumn{2}{|c|}{ 60-degree drill angle } & \multicolumn{2}{|c|}{ 90-degree drill angle } \\
\hline & Mean & Max. & Mean & Max. & Mean & Max. & Mean & Max. \\
\hline 1 & 0.91 & 1.67 & 0.81 & 1.95 & 1.08 & 2.87 & 0.98 & 2.45 \\
\hline 2 & 1.12 & 2.24 & 0.75 & 1.61 & 0.92 & 2.12 & 1.27 & 3.58 \\
\hline 3 & 1.12 & 1.75 & 0.90 & 2.88 & 1.76 & 2.57 & 1.56 & 5.11 \\
\hline 4 & 1.50 & 2.57 & 1.41 & 2.67 & 1.62 & 2.50 & 1.40 & 3.65 \\
\hline 5 & 0.97 & 1.41 & 1.29 & 2.30 & 1.46 & 2.53 & 1.15 & 4.59 \\
\hline 6 & 1.07 & 1.54 & 1.53 & 2.28 & 1.85 & 3.01 & 1.01 & 5.41 \\
\hline 7 & 0.89 & 1.84 & 1.16 & 2.67 & 1.24 & 2.72 & 1.36 & 4.36 \\
\hline 8 & 0.46 & 1.57 & 0.55 & 1.63 & 0.61 & 2.35 & 0.87 & 13.13 \\
\hline Mean (standard deviation) & \multicolumn{2}{|c|}{$1.01(0.294)$} & \multicolumn{2}{|c|}{$1.05(0.348)$} & \multicolumn{2}{|c|}{$1.32(0.433)$} & \multicolumn{2}{|c|}{$1.20(0.240)$} \\
\hline
\end{tabular}

TABLE A2. Mean force and standard deviation for each depth/velocity combination. All trials were performed in cortical bone with a 5-mm fluted burr, at a 20-degree drill angle, and a drill spindle speed of 80,000 RPM.

\begin{tabular}{|c|c|c|c|}
\hline Cutting depth (mm) & Cutting velocity $(\mathrm{mm} / \mathrm{s})$ & Mean force magnitude $(\mathrm{N})$ & Standard deviation $(\mathrm{N})$ \\
\hline 0.62 & 2.00 & 0.65 & 0.15 \\
\hline 0.62 & 4.00 & 0.90 & 0.11 \\
\hline 0.62 & 8.00 & 1.03 & 0.11 \\
\hline 1.00 & 1.00 & 0.90 & 0.27 \\
\hline 1.00 & 2.00 & 1.02 & 0.21 \\
\hline 1.00 & 4.00 & 1.32 & 0.17 \\
\hline 1.00 & 6.00 & 1.55 & 0.26 \\
\hline 1.33 & 1.00 & 1.28 & 0.42 \\
\hline 1.33 & 2.00 & 1.44 & 0.34 \\
\hline 1.33 & 4.00 & 1.95 & 0.35 \\
\hline 1.33 & 5.35 & 2.23 & 0.53 \\
\hline 1.64 & 1.00 & 1.82 & 0.22 \\
\hline 1.64 & 2.00 & 2.39 & 0.49 \\
\hline 1.64 & 4.00 & 2.78 & 0.33 \\
\hline
\end{tabular}

TABLE A3. Comparison of different cutting velocities at each depth of cut tested. All trials were performed in cortical bone with a 5-mm fluted burr, at a 20-degree drill angle, and a drill spindle speed of 80,000 RPM. In this table, "Velocity 1" and "Velocity 2"' are the 2 values being compared.

\begin{tabular}{|c|c|c|c|c|c|}
\hline Cutting depth (mm) & Velocity $1(\mathrm{~mm} / \mathrm{s})$ & Velocity $2(\mathrm{~mm} / \mathrm{s})$ & Mean force difference $(1-2)(N)$ & Standard error $(\mathrm{N})$ & $p$ \\
\hline \multirow[t]{3}{*}{0.62} & 2.0 & 4.0 & -0.25 & 0.06 & 0.002 \\
\hline & 2.0 & 8.0 & -0.38 & 0.06 & $<0.001$ \\
\hline & 4.0 & 8.0 & -0.13 & 0.06 & 0.137 \\
\hline \multirow{6}{*}{1.00} & 1.0 & 2.0 & -0.12 & 0.12 & 1.000 \\
\hline & 1.0 & 4.0 & -0.42 & 0.12 & 0.007 \\
\hline & 1.0 & 6.0 & -0.64 & 0.12 & $<0.001$ \\
\hline & 2.0 & 4.0 & -0.30 & 0.12 & 0.092 \\
\hline & 2.0 & 6.0 & -0.52 & 0.12 & 0.001 \\
\hline & 4.0 & 6.0 & -0.23 & 0.12 & 0.370 \\
\hline \multirow[t]{6}{*}{1.33} & 1.0 & 2.0 & -0.16 & 0.21 & 1.000 \\
\hline & 1.0 & 4.0 & -0.66 & 0.21 & 0.022 \\
\hline & 1.0 & 5.35 & -0.94 & 0.21 & 0.001 \\
\hline & 2.0 & 4.0 & -0.51 & 0.21 & 0.135 \\
\hline & 2.0 & 5.35 & -0.78 & 0.21 & 0.005 \\
\hline & 4.0 & 5.35 & -0.28 & 0.21 & 1.000 \\
\hline \multirow[t]{3}{*}{1.64} & 1.0 & 2.0 & -0.56 & 0.18 & 0.016 \\
\hline & 1.0 & 4.0 & -0.95 & 0.18 & $<0.001$ \\
\hline & 2.0 & 4.0 & -0.39 & 0.18 & 0.133 \\
\hline
\end{tabular}


TABLE A4. Comparison of different cutting depths at each of the velocities tested. All trials were performed in cortical bone with a 5-mm fluted burr, at a 20-degree drill angle, and a drill spindle speed of 80,000 RPM. In this table, "Depth 1 " and

"Depth 2" are the 2 values being compared. For all comparisons, a Dunnett C test was used after the analysis of variance test because of inhomogeneous variances and thus exact $p$ values not determined.

\begin{tabular}{|c|c|c|c|c|c|}
\hline Cutting velocity $(\mathrm{mm} / \mathrm{s})$ & Depth $1(\mathrm{~mm})$ & Depth $2(\mathrm{~mm})$ & Mean force difference $(1-2)(\mathrm{N})$ & Standard error $(\mathrm{N})$ & $p$ \\
\hline \multirow[t]{3}{*}{1.0} & 1.00 & 1.33 & -0.38 & 0.18 & $>0.05$ \\
\hline & 1.00 & 1.64 & -0.92 & 0.12 & $<0.05$ \\
\hline & 1.33 & 1.64 & -0.54 & 0.17 & $<0.05$ \\
\hline \multirow[t]{6}{*}{2.0} & 0.62 & 1.00 & -0.37 & 0.09 & $<0.05$ \\
\hline & 0.62 & 1.33 & -0.79 & 0.13 & $<0.05$ \\
\hline & 0.62 & 1.64 & -1.74 & 0.18 & $<0.05$ \\
\hline & 1.00 & 1.33 & -0.42 & 0.14 & $>0.05$ \\
\hline & 1.00 & 1.64 & -1.36 & 0.19 & $<0.05$ \\
\hline & 1.33 & 1.64 & -0.94 & 0.21 & $<0.05$ \\
\hline \multirow{6}{*}{4.0} & 0.62 & 1.00 & -0.42 & 0.07 & $<0.05$ \\
\hline & 0.62 & 1.33 & -1.04 & 0.13 & $<0.05$ \\
\hline & 0.62 & 1.64 & -1.88 & 0.12 & $<0.05$ \\
\hline & 1.00 & 1.33 & -0.63 & 0.14 & $<0.05$ \\
\hline & 1.00 & 1.64 & -1.45 & 0.13 & $<0.05$ \\
\hline & 1.33 & 1.64 & -0.83 & 0.17 & $<0.05$ \\
\hline
\end{tabular}

